\title{
Future Prospects for IgA Nephropathy
}

IgA nephropathy has the highest incidence among the various chronic glomerulonephritides in Japan. IgA nephropathy is assumed to be an immunecomplex-mediated glomerulonephritis, and the initiating and progressive factors involved in this disease have gradually been indentified. However, it is necessary to determine whether the etiology of $\operatorname{IgA}$ nephropathy is antigendependent or antigen-independent. Although there is no specific treatment for patients with $\operatorname{IgA}$ nephropathy at present, more trials of new treatments including multicenter studies are warranted for such patients. Further examinations are also needed for better understanding of the pathogenesis in order to hasten the development of specific therapy for this disease. 